[3] Hakkim A, Fürnrohr BG, Amann K, Laube B, Abed UA, Brinkmann V, et al. Impairment of neutrophil extracellular trap degradation is associated with lupus nephritis. Proc Natl Acad Sci U S A. 2010;107(21):9813-8.

Disclosure of Interest: None declared

DOI: 10.1136/annrheumdis-2017-eular.4879

\section{THU0275 IMPORTANCE OF SERUM TITERS OF ANTIBODIES AGAINST DOMAIN 1 OF B2 GLYCOPROTEIN 1}

$\underline{\text { J.A. Gómez-Puerta }}^{1,2}$, T. Urrego ${ }^{2}$, A. Hernández ${ }^{3}$, S. Ruiz Giraldo ${ }^{3}$, C. Rua ${ }^{4}$, J. Duque Botero $^{3}$, M. Velázquez ${ }^{5}$, A.P. Cadavid ${ }^{5}$, A.L. Vanegas-García ${ }^{1,6}$, C.H. Muñoz ${ }^{1,6}$, L.A. González ${ }^{1}$, G. Vásquez ${ }^{1,2}$. ${ }^{1}$ Grupo de Reumatología; ${ }^{2}$ Grupo de Inmunología Celular e Inmunogenética; ${ }^{3}$ Departamento de Medicina Interna; ${ }^{4}$ Grupo de Investigación en Trombosis; ${ }^{5}$ Grupo de Reproducción, Universidad de Antioquia; ${ }^{6}$ Grupo de Reumatología, Hospital San Vicente Fundación, Medellín, Colombia

Background: There is an increasing interest in the study of domain reactivity of antibodies against $\beta 2$ glycoprotein I (anti-B2GPI) in patients with antiphospholipid syndrome (APS). Antibodies targeting domain 1 of the molecule (anti-D1) as the most relevant autoantibody subpopulation. Previous information of anti-D1 B2GPI comes from APS cohorts with Caucasian, Asian, and African-American patients. However, there are no studies in Mestizo and Afro-Latin American patients.

Objectives: Our aim was to evaluate the prevalence of Anti-D1 B2GPI antibodies in a cohort of Colombian patients with systemic lupus erythematosus (SLE) with and without thrombosis, primary APS and patients with previous history of recurrent miscarriages $(\mathrm{RM})$ without APS criteria.

Methods: In this cross-sectional study we measured Anti-D1 B2GPI antibodies in a group of patients from Rheumatology Department, Coagulation clinic and Recurrent Pregnancy Loss Program at the Reproduction Group at Hospital San Vicente Fundación and Universidad de Antioquia, respectively, at Medellín, Colombia. Anti-D1 B2GP1 antibodies were tested using a chemiluminescent immunoassay (QUANTA Flash B2GPI IgG, Inova Diagnostics). Mann-Whitney tests were used to compare data.

Results: One hundred and seventy seven (median age $33.5 \pm 12.1$ years; $89 \%$ women) patients were included. One hundred thirty eight patients had SLE (78\%), 27 primary APS (15\%) and $13 \mathrm{RM}(7 \%)$. Fifty five (31\%) out of 177 patients had history of thrombosis and $41(23 \%)$ of pregnancy losses. Overall, Anti-D1 B2GPI antibodies were positive (>20 CU) in $35(20 \%)$ of patients. Anti-D1 B2GPI were positive in $23 \%, 17 \%$ and $0 \%$ of patients with primary APS, SLE and RM, respectively. Overall, serum Anti-D1 B2GPI were significantly higher in patients with than without previous thrombosis $(149.1 \pm 336.1$ vs $16.3 \pm 61.8 \mathrm{CU}, p<0.0001)$ and in patients (SLE or primary APS) with previous history of pregnancy losses (40.2 \pm 123.1 vs $21.0 \pm 74.5 \mathrm{CU}, \mathrm{p}=0.04)$. Anti-D1 B2GPI were significantly higher in patients with primary APS vs SLE with thrombosis, and in patients with SLE with thrombosis vs SLE without thrombosis (Figure). No clinical associations were found among Anti-D1 B2GPI antibodies and other APS features.

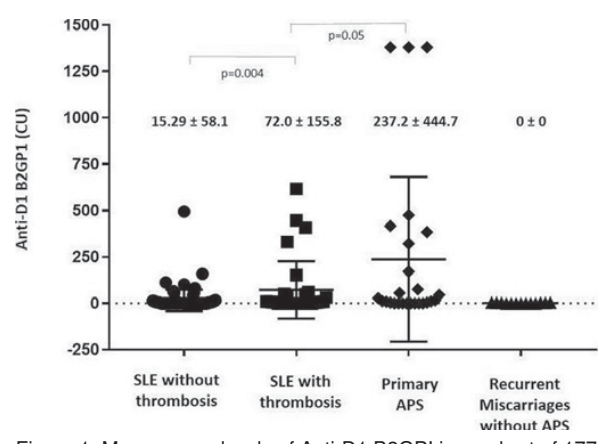

Figure 1. Mean serum levels of Anti-D1 B2GPI in a cohort of 177 patients.

Conclusions: Serum titers of Anti-D1 B2GPI antibodies were more than 9 times and 2 times higher in patients with thrombosis and pregnancy losses, respectively. In addition, serum titers were significantly higher in patients with primary APS than in SLE patients with thrombosis. Whether Anti D1- B2GPI antibodies titers are useful to differentiate patient with primary and secondary APS requires further analysis.

Acknowledgements: JA Gómez-Puerta was supported by Colciencias (conv. 656 de 2014). Anti-D1 B2GPI antibodies were provided by Inova, Werfen, Colombia

Disclosure of Interest: None declared

DOI: 10.1136/annrheumdis-2017-eular.2767

\section{THU0276 INTERFERON-RELATED CHEMOKINE IP-10 IS A POTENTIAL BIOMARKER IN INCOMPLETE SYSTEMIC LUPUS ERYTHEMATOSUS}

W. Lambers ${ }^{1}$, K. De Leeuw ${ }^{1}$, M. Jonkman ${ }^{2}$, H. Bootsma ${ }^{1}$, J. Westra ${ }^{1}$. ${ }^{1}$ Rheumatology and Clinical Immunology; ${ }^{2}$ Dermatology, UMCG, Groningen, Netherlands

Background: Incomplete SLE (iSLE) is the designation of patients who display symptoms that are typical for SLE, but with insufficient criteria to fulfil the diagnosis. Unfortunately, predictive biomarkers for SLE development are lacking. Increased IFN-type I production is an important factor in the pathogenesis of SLE. Interferon-regulated chemokines therefore could possibly be useful as biomarkers for disease progression to SLE. Candidate biomarkers IFN- $\gamma$ induced protein 10 (IP-10) and monocyte chemo attractant protein (MCP-1) have shown to be increased in SLE and to correlate with disease activity.

Objectives: To determine possible candidate biomarkers IFN- $\gamma$ induced protein 10 (IP-10) and monocyte chemo attractant protein (MCP-1) in patients with iSLE. Methods: Serum samples were collected of 30 iSLE patients, 29 SLE patients, and 17 ANA-negative patients with histologically proven cutaneous lupus erythematosus (ANCLE). Outcomes were compared with 25 age- and gender-matched controls (CTL) and 31 rheumatoid arthritis (RA) patients as disease control group. Levels of IP-10 and MCP-1 were measured using ELISA.

Results: IP-10 was significantly increased in SLE and RA patients compared with CTL and ANCLE. IP-10 levels were increased in 23\% of iSLE patients. These patients had higher SLE disease activity index (SLEDAI) and more frequently decreased C3 level and joint involvement compared to those with normal IP-10 levels. Regarding MCP-1 levels, no significant differences were found between any of the groups and no correlations with clinical markers was found.

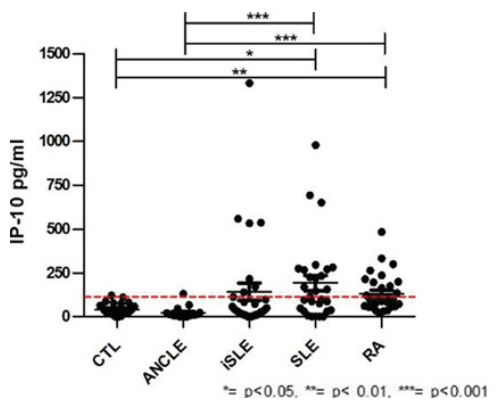

Conclusions: Levels of IP-10, but not MCP-1, might be useful as a predictive biomarker for progression to SLE in patients with iSLE, although future prospective longitudinal analyses are needed to confirm this hypothesis.

Disclosure of Interest: None declared

DOI: 10.1136/annrheumdis-2017-eular.4016

\section{THU0277 REASONS FOR DEFICIENCY OF PHYSICAL INACTIVITY IN PATIENTS WITH SYSTEMIC LUPUS ERYTHEMATOSUS}

L. Lipovskiy, C. Baerwald, O. Seifert. Rheumatology Unit, Department of Internal Medicine, University Hospital Leipzig, Leipzig, Germany

Background: Systemic lupus erythematosus (SLE) is associated with musculoskeletal complaints, fatigue and reduced quality of life. Physical exercise can play a crucial role in the treatment of rheumatic diseases, optimizing both physical and mental health.

Objectives: To characterize physical activity (PA) and its impact on SLE patients. Methods: 85 SLE outpatients (92\% female; age $44.2 \pm 12.5 ; 57.5 \%$ with SLEDAI score $\leq 5,42.5 \%$ with SLEDAI score $\geq 6$ ) were included in this study. The following questionnaires were utilized: painDETECT, visual analog scale for pain (VAS 0 - $100 \mathrm{~mm}$ ), short-form health survey (SF - 36, quality of life), the FACIT-Fatigue Scale and the Health Assessment Questionnaire (HAQ - DI). PA was assessed for every patient using the long form of International Physical Activity Questionnaire (IPAQ - LF) and the Metabolic Equivalent of Task (MET) minutes per week (min/wk) (physiological measurement expressing calories of physical activities). The participants were classified in 3 groups according to the PA levels conferring to the guidelines of IPAQ. Physical inactivity was defined as fewer than $150 \mathrm{~min} /$ week spent in moderate or vigorous physical activities. Furthermore, the patient reasons "to be not physical active" and their opinion regarding the influence of PA on disease-related symptoms (on a scale of $0-10$ ) were obtained.

Results: $10.6 \%$ of SLE patients were physically inactive $(525.2 \pm 277.3$ METMin/wk), $31.8 \%$ had a moderate physical activity, and $57.6 \%$ were physically active. Physical inactivity was associated with higher fatigue (FACIT-F, $p<0.04$ ) and lower "vitality" (SF-36, $\mathrm{p}<0.03)$ scores. Moreover, the subjective impact of fatigue on PA was significantly higher in physically inactive patients compared to physically active patients (the patient's score: $7.75 \pm 1.25$ vs. $5.5 \pm 0.94, p<0.04$ ). Moderate and severe pain (more than $40 \mathrm{~mm}$ VAS) was also associated with physical inactivity (OR $12.38,95 \% \mathrm{Cl} 1.69$ to $144.3, \mathrm{p}=0.0056)$. In contrast, HAQ-DI was not related to levels of PA. Although physical inactivity correlated 